survival, highlight the potential for the continuing spread of the epidemic and the need for more health promotion initiatives that have been shown to be effective.

We thank the Camden and Islington Health Authority for its financial support. We thank Dr Andrew Copas for statistical advice. We also thank members of the working group.

Contributors: AN, DEM, and AMJ initiated and designed the study. JPD and AN managed the project. JPD analysed the data, wrote the first draft, and coordinated subsequent revisions of the paper. All authors contributed to the final version of this paper. JPD and DEM are the guarantors.

Funding: Camden and Islington Health Authority.
Competing interests: None declared.

1 Communicable Disease Surveillance Centre. AIDS and HIV infection in the UK: monthly report. Commun Dis Rep CDR Wkly 1999;9:121-2.

2 From the Centers for Disease Control and Prevention. Increases in unsafe sex and rectal gonorrhea among men who have sex with men-San Francisco, California, 1994-1997. JAMA 1999:281:696-7.

3 Nardone A, Mercey DE, Johnson AM. Surveillance of high risk behaviour amongst homosexual men in a central London health authority Genitourin Med 1997;73:198-202

4 Nardone A, Dodds JP, Mercey DE, Johnson AM. Active surveillance of sexual behaviour among homosexual men in London. Commun Dis Public Health 1998; 1:197-201.

5 Sexually transmitted disease quarterly report: gonorrhoea in England and Wales. Commun Dis Rep CDR Wkly 1998;8:194-6.

(Accepted 22 February 2000)

\title{
Retrospective case note review of acute and inpatient stroke outcomes
}

\author{
Nabil Aly, Kevin McDonald, Michael Leathley, Anil Sharma, Caroline Watkins
}

The annual incidence of stroke in the community is about 2 per 1000 population, ${ }^{1}$ whereas among hospital inpatients it is 11 per $1000 .{ }^{2}$ However, a study that systematically and simultaneously identifies all inpatients experiencing stroke and all patients admitted with stroke does not exist. Previous work on stroke among inpatients has excluded some patients-for example, those with ${ }^{3}$ or without ${ }^{2}$ obvious iatrogenic predisposing factors. Similarly, although risk factors for stroke have been used as predictors of an event in the context of a study, ${ }^{3}$ these are often not documented clinically. Secondary prevention is dependent on identification and documentation of risk factors.

We compared outcomes and the identification and documentation of known risk factors in a cohort of patients admitted with a stroke or having had a stroke while in hospital (having been admitted to hospital with a primary diagnosis other than stroke).

\section{Subjects, methods, and results}

University Hospital Aintree serves a predominantly urban population of 250000 and admits about 32000 patients annually. Its stroke unit has 18 acute and 25 rehabilitation beds. Guidelines for the management of acute stroke are available throughout the hospital.
We identified all patients with a primary diagnosis of stroke (excluding transient ischaemic attacks and subarachnoid haemorrhages) on a stroke register. From October 1994 to March 1997, 100 inpatients with stroke and 1274 patients admitted with stroke were identified prospectively by a 24 hour, on-call stroke research team or retrospectively from the hospital discharge coding. Data collection was by retrospective review of case notes.

Median ages were 75 (interquartile range 67-82) years for inpatients and 74 (66-81) years for admitted patients. Fifty four (54\%) inpatients and $647(51 \%)$ admitted patients were female. Forty seven (47\%) inpatients and $537(42 \%)$ admitted patients were managed in the stroke unit.

The table shows the numbers of patients for whom known risk factors for stroke were clearly documented and the numbers for whom no documentation existed. According to documentation, cardiovascular risk factors were significantly higher in inpatients whereas previous strokes or transient ischaemic attacks were more common among admitted patients. Documentation was less complete for inpatients than for admitted patients.

Of the $80(80 \%)$ inpatients and $1092(86 \%)$ admitted patients who had computed tomography, $5(6 \%)$
Aintree Stroke Unit, Department of Medicine for the Elderly, University Hospital Aintree, Liverpool L9 7AL Nabil Aly specialist registrar Kevin McDonald research associate Michael Leathley research fellow

Anil Sharma consultant physician Caroline Watkins honorary research fellow

Correspondence to: A Sharma aksharma@ aintreestar. u-net.com

BMJ 2000;320:1511-2

Identification and documentation of known stroke risk factors among patients who had a stroke while in hospital and among patients who were admitted with stroke. Values are numbers (percentages) of patients

\begin{tabular}{|c|c|c|c|c|c|c|}
\hline & \multicolumn{3}{|c|}{ Identified } & \multicolumn{3}{|c|}{ Not documented } \\
\hline & Inpatients $(n=100)$ & Admitted patients ( $n=1274$ ) & $P$ value & Inpatients ( $n=100)$ & Admitted patients ( $n=1274$ ) & $P$ value \\
\hline Cardiac failure & $35(35)$ & $41(3)$ & $<0.01$ & $60(60)$ & $954(75)$ & $<0.01$ \\
\hline Atrial fibrillation & $32(32)$ & $253(20)$ & $<0.01$ & $38(38)$ & $223(18)$ & $<0.01$ \\
\hline Myocardial infarction & $25(25)$ & $181(14)$ & $<0.01$ & $30(30)$ & $207(16)$ & $<0.01$ \\
\hline Angina & $19(19)$ & $138(11)$ & $<0.03$ & $68(68)$ & $830(65)$ & $>0.61$ \\
\hline Hypertension & $31(31)$ & $480(38)$ & $>0.19$ & $30(30)$ & $196(15)$ & $<0.01$ \\
\hline Diabetes mellitus & $11(11)$ & $149(12)$ & $>0.88$ & $33(33)$ & $195(15)$ & $<0.01$ \\
\hline Previous stroke & $16(16)$ & $329(26)$ & $<0.04$ & $38(38)$ & $188(15)$ & $<0.01$ \\
\hline Previous transient ischaemic attack & $9(9)$ & $240(19)$ & $<0.02$ & $56(56)$ & $334(26)$ & $<0.01$ \\
\hline Ever smoked & 39 (39) & 685 (54) & $<0.01$ & $20(20)$ & $162(13)$ & $>0.05$ \\
\hline
\end{tabular}

All tests were with Yates's corrected $\chi^{2}$. 
and $144(13 \%)$ respectively had a primary intracerebral haemorrhage $\left(\chi^{2}=2.64, \mathrm{P}>0.05\right)$.

The inpatients remained in hospital longer after stroke (median 31 (interquartile range 13-59) days) than the admitted patients (16 (6-43) days). Twenty four (24\%) inpatients returned to their previous residence, compared with $799(63 \%)$ admitted patients (odds ratio $0.19,95 \%$ confidence interval 0.11 to 0.31 ). Sixteen (16\%) inpatients were newly discharged to an institution, compared with $124(10 \%)$ admitted patients (1.77, 95\% confidence interval 0.93 to 3.16 ), which may partly account for the longer stay for inpatients. Sixty $(60 \%)$ inpatients died in hospital, compared with 351 $(28 \%)$ admitted patients $(3.94,95 \%$ confidence interval 2.55 to 6.15$)$; stroke was the primary or secondary cause of death for $51(85 \%)$ inpatients and $301(86 \%)$ admitted patients.

\section{Comment}

Although the inpatients and the admitted patients were similar in terms of age and sex, inpatients stayed in hospital longer, were more likely to die in hospital, and had less well documented risk factors. Improving staff awareness on medical and surgical wards regarding the importance of the early identification and documentation of known risk factors for stroke may improve outcome.

We thank Liz Lightbody, Hazel Dickinson, and Dimitrios Theofanidis, who collected data from the European stroke database, and the BMJ reviewers (Gord Gubitz and M J Campbell) for their comments.

Contributors: NA contributed to the interpretation of the results and to writing the paper. $\mathrm{KMcD}$ analysed the data and contributed to writing the paper. ML advised on and contributed to the statistical analysis and interpretation of the results and to writing the paper. AS contributed to the interpretation of the results and to writing the paper and will act as guarantor for the paper. CW advised on the results, analysis, and interpretation and contributed to writing the paper.

Funding: None.

Competing interests: None declared.

1 Bamford J, Sandercock P, Dennis M, Warlow C, Jones L, McPherson K, et al. A prospective study of acute cerebrovascular disease in the community: the Oxfordshire community stroke project 1981-86: 1 . Methodology, demography and incident cases of first-ever stroke. $/$ Neurol Neurosurg Psychiatry 1988;51:1371-80.

2 Azzimondi G, Nonino F, Fiorani L, Vignatelli L, Stracciari A, Pazzaglia P, et al. Incidence of stroke among inpatients in a large Italian hospital. Stroke 1994;25:1752-4.

3 Mahaffey KW, Granger CB, Sloan MA, Thompson TD, Gore JM, Weaver WD, et al. Risk factors for in-hospital nonhemorrhagic stroke in patients with acute myocardial infarction treated with thrombolysis: results from GUSTO-I. Circulation 1998;97:757-64

(Accepted 5 April 2000)

\title{
Preventing dog bites in children: randomised controlled trial of an educational intervention
}

\author{
Simon Chapman, John Cornwall, Joanne Righetti, Lynne Sung
}

Department of Public Health and Community

Medicine,

University of

Sydney, Sydney,

NSW 2000,

Australia

Simon Chapman

associate professor

Delta Society

Australia, 6/3

Spring Street,

Sydney, NSW 2000,

Australia

John Cornwall

consultant

Joanne Righetti

consultant

Lynne Sung

consultant

Correspondence to: S Chapman

Simonc@pub.health. usyd.edu.au

BMJ 2000;320:1512-3

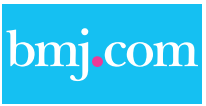

The CONSORT

checklist is

available on the

BMJ's website
Dog bites are a major cause of injury, particularly in children. ${ }^{12}$ Guidelines on prevention are generally unevaluated and include controls on high risk breeds, keeping dogs on a leash, animal training, and educating dog owners. ${ }^{3}{ }^{4}$ However, there are no evaluations of interventions designed to teach people how to avoid being attacked by a dog.

"Prevent-a-Bite" is an educational programme designed for primary school children. ${ }^{5}$ The programme aims to instil precautionary behaviour around dogs, assuming that this might reduce the incidence of attacks. A randomised controlled trial of the efficacy of the intervention was conducted in Australian children aged 7-8 years who were presented with an unsupervised opportunity to approach a strange dog.

\section{Participants, methods, and results}

Eight primary schools in metropolitan Sydney were randomly selected to participate in the trial. All agreed. The schools were cluster randomised into intervention and non-intervention control schools (four in each group), and two classes in each school were then selected to participate. Altogether 346 children aged 7-8 years took part. The study was approved by the human ethics committee of the University of Sydney.
The intervention consisted of a 30 minute lesson conducted by an accredited dog handler. The handler and dog demonstrated various "dos and don'ts" of behaviour around dogs, such as how to recognise friendly, angry, or frightened dogs and how children should approach dogs and owners when they wanted to pat a dog. Children practised patting the dog in the correct manner (that is, asking permission, approaching slowly, extending the hand palm down, patting the dog under the chin and on the chest, avoiding eye contact, walking away slowly and quietly) and precautionary and protective body posture to adopt when approached or knocked over by a dog. They were also told when not to disturb even a friendly, known dog (for example, when it is sleeping, eating, tied up, or in a car). A resource kit for teachers, which included activities to be undertaken before and after the demonstration, was also distributed.

Seven to 10 days after participating in the programme, children in the intervention schools were let out to play unsupervised in the school grounds. A docile Labrador dog was tethered five metres away from its owner, who was disguised as a tradesman. The children were not told that the dog was there and were videotaped by a hidden camera for 10 minutes. Children in control schools were let out to play in similar circumstances, but they had not received the intervention. 